\title{
Primera localización de un dormidero de milano real Milvus milvus L., 1758 en un eucaliptal en Álava.
}

\section{First localisation of a Red kite Milvus milvus L., 1758 roost in a eucalyptus wood in Alava.}

\author{
Antonio Sáenz de Santa María-Muniategui ${ }^{1 *}$, Gorka Belamendia-Cotorruelo ${ }^{1}$
}

*

\section{Resumen}

Durante el trabajo de campo del censo 2020-2021 para conocer la población de milano real (Milvus milvus) invernante en la península lbérica mediante el conteo en dormideros comunales, se observó la formación de uno en una pequeña repoblación de eucaliptos (género Eucalyptus) al noroeste de la provincia de Álava (País Vasco-España). Aunque el establecimiento de dormideros en este tipo de sustrato arbóreo es poco frecuente, nuestra observación sugiere la hipótesis de que los milanos empleen los eucaliptos si las condiciones atmosféricas son particularmente adversas. Asimismo, planteamos la urgente necesidad de conocer la distribución y ecología invernal de las poblaciones reproductoras ibéricas de milano real, cuyo estudio está dificultado por la llegada en invierno de multitud de conespecíficos de origen centro y norte-europeo.

Palabras clave: milano real, Milvus milvus, censo población invernal, dormidero comunal, eucaliptal, norte península Ibérica, Life Eurokite.

\begin{abstract}
\footnotetext{
${ }^{1}$ Aranzadi Zientzia Elkartea / Sociedad de Ciencias Aranzadi

* Correspondencia: asaenzsantamaria@gmail.com
}

During the 2020-2021 census to determine the wintering population of Red kite (Milvus milvus) in the Iberian peninsula by counting the number of communal roosts, one such roost was observed in a small reforested area of eucalyptus (genus Eucalyptus) in the northwest of the province of Álava (Basque Country-Spain). Although roosts located on this kind of arboreal substrate are rare, our observation suggests the hypothesis that Red kites will use eucalyptus if the atmospheric conditions are particularly adverse. Therefore, we propose that the distribution and winter ecology of Iberian breeding populations of Red 
kite be clarified with urgency, the study of which is hampered by the arrival of a multitude of conspecifics of central and northern-European origin in winter.

Key words: Red kite, Milvus milvus, winter population census, communal roost, eucalyptus forest, north Iberian Peninsula, Life Eurokite.

\section{Laburpena}

2020-2021 erroldako landa-lanean, Iberiar penintsulan negua igarotzen duen miru gorriaren (Milvus milvus) populazioa jakiteko etzaleku komunaletan egindako zenbaketaren bidez, Arabako ipar-mendebaldean (Euskal Autonomia Erkidegoa-Espainia) eukaliptoen (Eucalyptus generoa) basoberritze txiki batean, horietako baten sorrera ikusi zen.

Zuhaitz-substratu mota horretan etzalekuak ezartzea oso ohikoa ez den arren, gure behaketak ondorengo hipotesia iradokitzen du, alegia, miruek eukaliptoak erabiltzen dituztela baldintza atmosferikoak bereziki txarrak badira. Azkenik, adierazi nahi dugu premiazkoa dela miru gorriaren ugaltze-populazio iberiarren neguko banaketa eta ekologia ezagutzea. Izan ere, haien azterketa zaila da, neguan Ipar eta Erdialdeko Europatik konespezifiko ugari iristen baitira.

Gako hitzak: miru gorria, Milvus milvus, neguko populazioaren errolda, etzaleku komunala, eukalipto-saila, Iberiar penintsulako iparraldea, Life Eurokite.

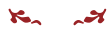

En enero de 2021 se llevó a cabo el primer censo invernal de dormideros de milano real (Milvus milvus) en Europa, coordinado por la Mitteleuropäische Gesellschaft zur Erhaltung der Greifvögel (Sociedad Centroeuropea para la Protección de las Rapaces), como parte del proyecto LIFE EUROKITE. Este programa busca censar los mismos dormideros de milano real todos los años hasta 2027 (LIFE EUROKITE, 2021). En el censo participan un total de 19 países entre los que destaca España al acoger el 62,4\% de la población invernante europea (Viñuela, 2012; LIFE EUROKITE, 2021).

En el País Vasco, la población invernante de milano real representa el 3\% del total ibérico (Deán, informe inédito 2021), acogiendo Álava el 56\% aproximadamente del total de ejemplares invernantes en esta comunidad autónoma (Belamendia y Elósegui, 2014). En esta provincia, los recuentos de su población invernante indican un incremento en el número de ejemplares contabilizados (Tabla 1). Al parecer, se trata de un incremento significativo que si bien en parte se debe a una mejora en la cobertura territorial del censo y a la mejora técnica de las capacidades de detección de ejemplares, también responde a un incremento de la propia población invernante (Belamendia y Elósegui, 2014).

Como en cualquier censo de esta especie, la metodología se basa en el seguimiento del vuelo de las aves en la distancia y en el conteo de los ejemplares posados en los dormideros, árboles en los que grandes grupos de aves se instalan para pasar la noche (Del Hoyo et al., 1994; De Juana y Valera, 2016; EBD, 2018; Viñuela et al. 1999). 


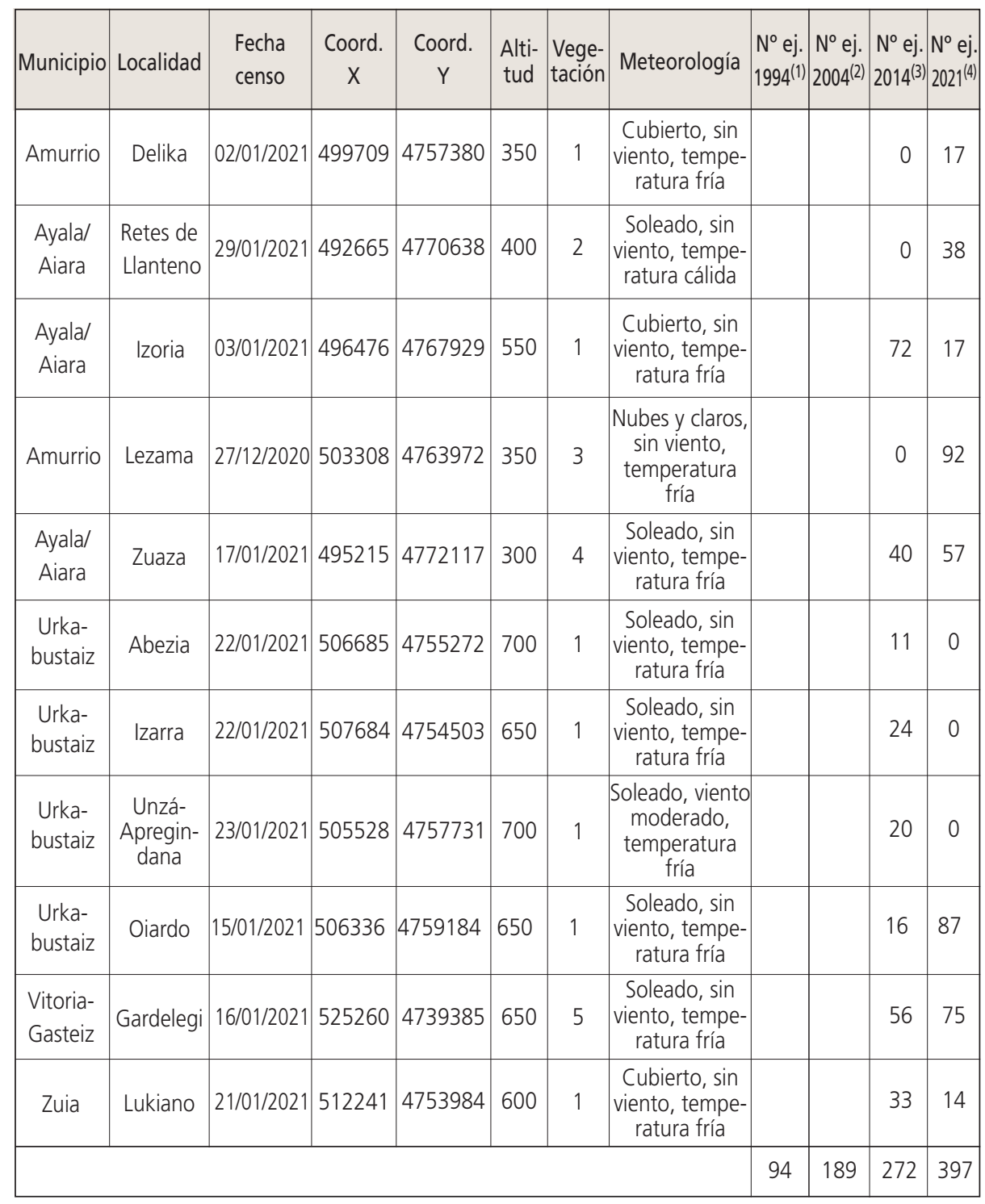

Tabla 1.- Evolución de la abundancia de milanos reales que invernan en la zona de estudio. Vegetación: (1) Quercus robur; (2) Quercus robur con Pinus sylvestris; (3) Pinus radiata; (4) Quercus robur con Pinus radiata y Eucalyptus sp.; (5) Pinus nigra.

Referencias: (1) Viñuela et al. 1999; (2) Viñuela 2012; (3) Belamendia y Elósegui 2014; (4) datos propios. Table 1.- Evolution of the abundance of Red kites that winter in the study area. Vegetation: (1) Quercus robur; (2) Quercus robur with Pinus sylvestris; (3) Pinus radiata; (4) Quercus robur with Pinus radiata and Eucalyptus sp.; (5) Pinus nigra.

References: (1) Viñuela et al. 1999; (2) Viñuela 2012; (3) Belamendia and Elósegui 2014; (4) own data. 
Generalmente, los ejemplares se posan previamente en uno o dos predormideros hasta que al filo de la oscuridad máxima, se instalan en el dormidero definitivo (EBD, 2018). Mientras dura la luz diurna e incluso durante el anochecer es común que todo el grupo levante el vuelo si le sobresalta algún sonido o presencia, volviendo al lugar en el que estuvieran en ese momento (Belamendia y Elósegui, 2014).

La localización del dormidero objeto de esta nota se sitúa en el municipio alavés de Ayala-Aiara, al suroeste del País Vasco y en el noroeste de la provincia de Álava, a una altitud de 325 msnm (Aiarako Udala, 2021). El área presenta una orografía de pequeñas montañas con amplios valles entre ellas (Aiarako Udala, 2021), que albergan un mosaico agroforestal en el que destacan manchas de bosques caducifolios nativos y plantaciones de pino y eucalipto, bordeados por espacios de campiña cantábrica de pasto de siega (Loidi et al., 2011). En esta zona, la expansión de las repoblaciones de Eucalyptus sp. presenta un ritmo elevado (HAZI, 2020), pasando de ocupar $225 \mathrm{Ha}$ en el año 2010 a 1.342 Ha en el año 2020 (Inventario Forestal 2020. HAZI, 2020).

La climatología es templada y húmeda sin estación seca (Euskalmet, 2011), propicia para la dedicación agroganadera con ganado vacuno y ovino que utiliza para su alimentación la campiña (Cendrero, 1986). Es una zona muy antropizada con una población bastante dispersa y una densidad media de 21,07 hab/ $\mathrm{km}^{2}$ (EUSTAT, 2021).

El 26 de diciembre del 2020, con tiempo soleado y en calma, se efectuó una primera visita al dormidero tradicional (conocido desde hace años), ubicado sobre ejemplares de Quercus robur que forman bosquete con pinos de Monterrey (Pinus radiata) y eucaliptos. Días después, el 17 de enero de 2021, durante un segundo censo, las condiciones climatológicas habían cambiado debido a una importante inestabilidad acontecida en la zona por una gran nevada que cubrió toda el área. Aquel día, los milanos reales comenzaron a aproximarse al dormidero desde primeras horas de la tarde. Hacia las 15:00 horas (UTC+1) empezaron a entrar a los predormideros, pero el viento soplaba fuerte y no acababan de posarse. Hacia las 17:00 horas (UTC+1) empezaron a instalarse entre el follaje de unos eucaliptos y para las 18:00 horas (UTC+1) estaban todos apostados allí. Allí permanecieron cuando la oscuridad se hizo completa, lo que implica que las aves debieron pasar la noche en los eucaliptos.

Aunque es la primera vez que se localiza en Álava un dormidero sobre este tipo de arbolado, hay citas para otras áreas de la península Ibérica como Cantabria, Badajoz, Asturias, Bizkaia-Vizcaya y Andalucía (Consejería del Medio Ambiente y Ordenación del Territorio, Junta de Andalucía 2016; Deán, informe inédito 2021) o incluso en países de nuestro entorno como Marruecos (Radi et al., 2020) o Portugal (Ferreira et al., 2014-2015).

A pesar de este leve servicio ecosistémico que prestan los eucaliptales, están claros los efectos ambientales generalmente nocivos que tienen sus plantaciones (Elósegui et al., 2020). Asimismo, son muchas las amenazas de origen antrópico que se ciernen sobre esta ave (veneno, atropellos, caza, colisiones tendidos eléctricos) (Viñuela et al., 1999; Viñuela, 2012; Belamendia y Elósegui, 2014), por lo que se hace especialmente importante la continuidad de estos censos (Hildén et al., 1988). 


\section{Conclusiones provisionales}

$1^{\text {a. }}$ Parece evidente que en esta zona los milanos reales invernantes pueden establecer dormideros entre el follaje de los eucaliptos.

$2^{a}$. Aunque estos dormideros reúnen unas condiciones mejores de aislamiento y son similares en altura respecto a los dormideros en quercíneas y coníferas, en esta área tienen un carácter secundario y marginal (Figura 1).

$3^{a}$. Este carácter subóptimo puede significar que sólo se establecen si las condiciones atmosféricas son adversas y, por lo tanto, requieren de una mayor protección, dado el carácter perennifolio de los eucaliptos.

$4^{a}$. Sería de interés hacer un seguimiento del sustrato arbóreo de los dormideros para aceptar o rechazar esas hipótesis o plantear otras.

$5^{a}$. Las declaraciones como especie "en peligro de extinción" en el Catálogo Español de Especies Amenazadas (Real Decreto 139/2011) y la calificación desde el año 2013 como especie "en peligro" en el Catálogo Vasco de Especies Amenazadas (Orden de 18 de junio de 2013, de la Consejera de Medio Ambiente y Política Territorial del Gobierno Vasco) hacen referencia a la especie como reproductora. En consecuencia, creemos necesario realizar estudios activos, y no meros conteos, que investiguen la distribución y ecología invernal que muestran los milanos reales reproductores ibéricos, dado que nuestro conocimiento fuera de la época reproductiva está dificultado precisamente por la llegada de aves centro y norte-europeas.

$6^{a}$. En general, la población invernante de milano real ha aumentado en Álava así como en la Comunidad Autónoma Vasca.

Sustratos forestales de dormideros del milano real. Álava 2021.

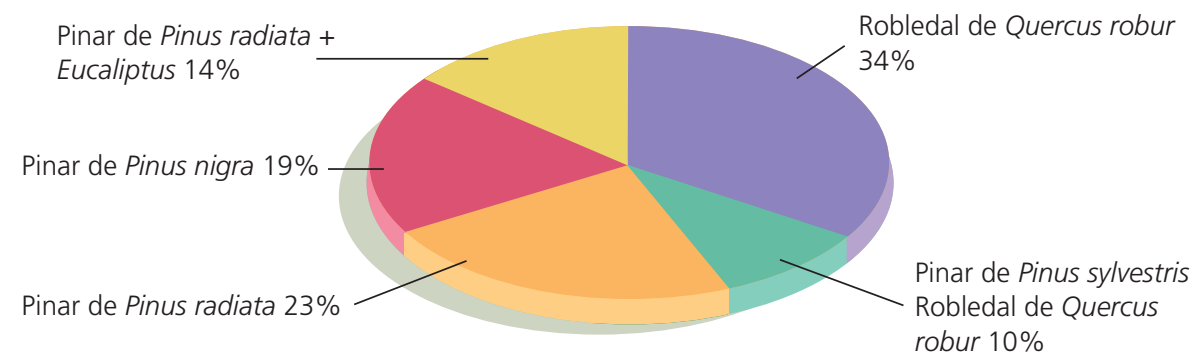

Fig. 1.- Sustratos forestales sobre los que se asientan los dormideros de milano real en Álava. Los porcentajes se corresponden con el número de ejemplares que utiliza cada uno de los sustratos arbóreos respecto al total del censo 2021 (Fuente: datos propios).

Fig. 1.- Forest substrates on which the roosts of Red kite in Álava are located. The percentages correspond to the number of specimens used by each of the tree substrates with respect to the total of the 2021 census (Source: own data). 


\section{Agradecimientos}

Los autores agradecen a Estitxu Breñas González de Zárate por su versión al euskera y a Estíbaliz Kapetillo por su aportación al inglés.

Asimismo, agradecen a Juan Ignacio Deán Pinedo, de la Sociedad de Ciencias Gorosti, por su sugerencia sobre el interés del tema.

A continuación, desean agradecer a los dos revisores anónimos que con sus comentarios han contribuido a mejorar la versión inicial de esta Nota y hacer constar que los comentarios del editor han mejorado sustancialmente el resultado final y la claridad de la misma.

\section{Bibliografía}

Aiarako Udala. Ayuntamiento de Ayala, 2021. Disponible en: http://www.aiarakoudala.com. (Acceso 4.02.2021).

Belamendia, G., Elósegui, R., 2014. Censo de Milano real (Milvus milvus) invernante en Euskadi (Invierno 2013-2014). SEO/BirdLife, Madrid.

Cendrero, A., 1986. El Sector Agropecuario en el País Vasco. $3^{\circ}$ Curso Superior de Geografía de Euskal-Herria. INGEBA Instituto Geográfico del País Vasco, San Sebastián.

Consejería de Medio Ambiente y Ordenación del Territorio, 2016. Plan de Recuperación de Aves necrófagas. Seguimiento población milano real en 2015. Junta de Andalucía. Disponible en: https://www.juntadeandalucia.es/medioambiente/portal/home?category$\mathrm{Val}=$. (acceso 3-02-2021).

De Juana, E., Varela, J.M., 2016. Aves de España. Lynx-SEO/BirdLife, Barcelona.

Deán, J.I., 2021. Censo de Milano Real invernante en España. Enero 2020 y consolidación de resultados 2014-2020. Informe inédito. Sociedad de Ciencias Naturales Gorosti, Pamplona.

Del Hoyo, J., Elliot, A., Saragatal, J. (Eds.), 1994. Handbook of the Birds of the World. Vol. 2. New World Vultures to Guineafowl, Barcelona.

EBD. Estación Biológica de Doñana. ICTS-CSIC, 2018. Protocolo para el censo de dormideros de rapaces invernantes en el Parque Nacional de Doñana. Disponible en: http://icts.ebd.csic.es/documents. (Acceso 03-02-2021).

Elósegui A., Cabido C., Larrañaga A., Arizaga, J., 2020. Efectos ambientales de las plantaciones de eucaliptos en Euskadi y la península Ibérica. Munibe, Cienc. Nat. 68, 111-136.

Euskalmet, 2011. Climatología del País Vasco. Dirección de Atención a Emergencias y Meteorología. Eusko Jaurlaritza-Gobierno Vasco. (Acceso Web 03-02-2021).

EUSTAT, 2021. Instituto Vasco de Estadística-Euskal Estatiska Erakundea. Ayala-Aiara. (Acceso Web 04-02-2021). 
Fernández-García, J.M., Gracianteparaluceta, A., 2017. Censo en dormideros de milanos reales invernantes en Gipuzkoa. Año 2017. EFA 089/15 POCTEFA Ecogyp. Diputación Foral de Gipuzkoa-Gipuzkoako Foru Aldundia.

Ferreira R., Zina H., Marques A.T., Delgado A., Venade, D., Costa, H. (2014-2015). Wintering population of Red kite (Milvus milvus) in the Castro Verde SPA. (Portugal). Airo 23, 55-60.

HAZI, 2021. El bosque vasco en cifras 2020. Informe de HAZI Fundazioa sobre el inventario forestal vasco de 2020. (Acceso Web 21-05-2021).

Hildén, O., Koskimes, P., Sharrock, J.T.R., 1988. The study of long-term trends in distribution and numbers of birds by means of questionnaries. Ardeola 35(1), 15-30.

Inventario Forestal 2020. Mapa Forestal CAE 2020. Departamento de agricultura, pesca y política alimentaria. Departamento de desarrollo económico, sostenibilidad y medio ambiente. Eusko Jaurlaritza- Gobierno Vasco, Vitoria-Gasteiz, 2021.

LIFE EUROKITE, 2021. Results of the 1st LIFE EUROKITE Winter Count of 267 selected regulary counted Red Kite roosting sites in whole Europe. 08.01--10.01.2021 (extended to 02.01 -23.01.2021). Impact monitoring of the EUROKITE Project. Informe inédito - LIFE EUROKITE Project "Cross-border proection of the Red Kite in Europe by reducing humancaused mortality".

Loidi, J., Biurrun, I., Campos, J.A., García-Mijangos, I., Herrera, M., 2011. La vegetación de la Comunidad Autónoma del País Vasco. Leyenda del mapa de serie de vegetación a escala 1:50.000. Departamento de Medio Ambiente, Planificación Territorial, Agricultura y Pesca. Eusko Jaurlaritza-Gobierno Vasco, Vitoria-Gasteiz.

Radi, M., Iglesias, J.J., Khalimchi, R., Martín, J., Rousselon, K., Lorcher, F., Belamine, M., Torralvo, C., Fajardo, B., Fajardo, I., Garrido, R., 2020. New roosting site of Red Kite Milvus milvus in the province of Ifrane (Morocco). Go-South Bull. 17, 4-7.

Viñuela, J., 2012. Milano real (Milvus milvus). En: SEO/BirdLife: Atlas de las aves en invierno en España 2007-2010., 164-165. Ministerio de Agricultura, Alimentación y Medio Ambiente-SEO/BirdLife, Madrid.

Viñuela, J., Martí, R., Ruiz, A., 1999. El Milano Real en España. Monografía n. 6. SEO/ BirdLife, Madrid.

\section{常}

Fecha de recepción/ Date of reception: 12/02/2021

Fecha de aceptación / Date of acceptance: 19/06/2021

Editor Asociado / Associate editor: Iván de la Hera 